\title{
Application of aromatization catalyst in synthesis of carbon nanotubes
}

\author{
SONG RONGJUN ${ }^{1,2 *}$, YANG YUNPENG ${ }^{1,2}$, JI QING $^{1,2}$ and LI BIN ${ }^{1,2}$ \\ ${ }^{1}$ Department of Chemistry, College of Science, Northeast Forestry University, Harbin 150040, China \\ ${ }^{2}$ Heilongjiang Key Laboratory of Flame Retarded Polymeric Materials, Education Department of Heilongjiang Province, \\ Heilongjiang, China
}

MS received 17 January 2009; revised 30 January 2011

\begin{abstract}
In a typical chemical vapour deposition (CVD) process for synthesizing carbon nanotubes (CNTs), it was found that the aromatization catalysts could promote effectively the formation of CNT. The essence of this phenomenon was attributed to the fact that the aromatization catalyst can accelerate the dehydrogenation-cyclization and condensation reaction of carbon source, which belongs to a necessary step in the formation of CNTs. In this work, aromatization catalysts, H-beta zeolite, HZSM-5 zeolite and organically modified montmorillonite (OMMT) were chosen to investigate their effects on the formation of multi-walled carbon nanotubes (MWCNTs) via pyrolysis method when polypropylene and 1-hexene as carbon source and $\mathrm{Ni}_{2} \mathrm{O}_{3}$ as the charring catalyst. The results demonstrated that the combination of those aromatization catalysts with nickel catalyst can effectively improve the formation of MWCNTs.
\end{abstract}

Keywords. Carbon nanotube; aromatization reaction; nickel.

\section{Introduction}

The carbon nanotubes (CNTs), by virtue of their curved graphitic structure, small diameter (1 $\mathrm{nm}$ to $\leq 100 \mathrm{~nm}$ ), and high aspect ratio, possesses many appealing properties including semiconducting or metallic electrical behaviour, high mechanical strength, and interesting chemical and surface properties (Height et al 2005). Potential applications include mechanical actuators (Landi et al 2002), electronics (Avouris 2002), catalysis (Li et al 2002), sensors (Wood et al 2000), high-strength composites (Thostenson et al 2001), and adsorbents (Dillon and Heben 2001). While, in order to obtain a larger number of CNTs with desired material properties in a real-world commercial use, a cost-effective method was still required to fabricate it. Therefore, how to synthesize CNTs efficiently has always been under significant investigation by a number of R\&D groups since its first observation using electron microscope by Iijima (1991).

CNTs can be fabricated with exactly 12 pentagons and millions of hexagons of carbon, which belongs to the architecture of $s p^{2}$ bonded carbon and the subtlety of a certain group of topological defects that can create unique, closed cylindrical structures out of the planar graphite sheets (Kiang et al 1995; Dresselhaus et al 1996; Ajayan and Ebbesen 1997; Ebbesen 1997; Yakobson and Smalley 1997; Saito et al 1998; Ajayan 1999). Similarly, the aromatics i.e. benzene, toluene and naphthalene etc, also comprised of carbon hexagons. Especially for polycyclic aromatics hydrocarbons (PAHs), it almost contains a small planar graphite sheet in its structure. Catalyst of aromatization can act as an effective

\footnotetext{
*Author for correspondence (dew741105@163.com)
}

additive catalyst during the process of synthesizing CNTs. Up to now, to our best knowledge, there are no studies concerning this field. In previous reports, it was found that the mixture of polypropylene (PP) supported nickel catalyst (NiCat) which could be highly transformed into multi-walled carbon nanotubes (MWCNTs) by addition of organically modified montmorillonite (OMMT) via combustion reaction (Tang et al 2005a, b). After further investigation, it was found that the PP degradation reactions induced by the carbenium ions which was located on the surface or interlayer of montmorillonite containing hydrogen proton (H-MMT) derived from OMMT strongly influenced carbonization behaviours of PP (Song et al 2007). However, during the above degradation reactions, the aromatization reaction of degradation products belongs to a kind of important reaction induced by the carbenium ions. Thus the essence of high catalysis efficiency of OMMT/ $\mathrm{Ni}_{2} \mathrm{O}_{3}$ co-catalyst maybe attributed to the hydrocarbon aromatization reaction catalyzed by H-MMT during the pyrolysis of PP.

Based on the above hypothesis that aromatization reaction of carbon source can promote CNT formation, a hypothesis seems reasonable that the aromatics should be a type of the most suited carbon source for synthesizing CNTs. However, this is not true, up to now, except benzene, toluene and xylene, no one used other aromatics as carbon source during chemical vapour deposition (CVD) method to synthesize CNTs. The reason could be explained as follows. Obviously, the CNTs, as a curved graphitic structure carbon, its formation process should be different with other graphitic structure carbon. In previous papers, Reilly and co-workers demonstrated that free radical condensates (FRCs) is an intermediate for producing some forms of carbon such as soot and 
fullerenes (Reilly et al 2000a, b; Rodgers et al 2003, 2004; Reilly and Whitten 2006). In FRC chemistry, PAHs have some interesting properties that make them as ideal initiators for the nucleation of FRCs, which means that aromatics can act as ideal carbon source to synthesize soot, fullerenes, amorphous carbon etc. As for the CNTs, on the one hand, the whole carbonization reactions of CNT formation in a CVD process take place on the surface of certain metals, and each process needs to be catalyzed by certain metal (Stein and Fahr 1985). So if the aromatics are used as carbon source to synthesize CNTs, they will be more easily transformed into other forms of carbon such as soot, fullerenes and amorphous carbon etc, instead of the CNTs; on the other hand, as a kind of graphitic structure carbon, the CNTs formation process should also be similar with other graphitic structure carbon. According to the structure of CNT, the dehydrogenationcyclization reaction should belong to a necessary step to synthesize CNT. At the same time, the condensation reaction should be another necessary step due to long cylindrical graphite sheets structures. However, similarly, both of the processes also take place during the aromatization reaction of a typical hydrocarbon. Therefore, although the aromatics is not a good carbon source for synthesizing CNT, the combination of the aromatization catalyst and the catalyst used for CNT formation would result in high efficient transformation of the CNTs from a typical hydrocarbon in the CVD method.

In this report, three kinds of aromatization catalyst, i.e. Hbeta zeolite (large pore and strong acidity molecular sieve), HZSM-5 zeolite (medium pore and weak acidity molecular sieve), and OMMT (layered silicates), chosen and combined, respectively with nickel catalyst were applied to investigate their effects on the formation of MWCNTs from PP or 1hexene via pyrolysis. To date, as for CNT formation from hydrocarbons, it is widely accepted that the molecules with lower carbon number are more easily in situ catalysed to form MWNTs and relatively lower molecular hydrocarbon as carbon source is often in favour of perfect graphitization of CNTs. It is well known that the acidity of zeolite can result in the cracking reaction of bulky molecules under high temperature condition. Thus, it is reasonable that much more low molecular hydrocarbon should be produced from PP under high temperature condition in the presence of H-MMT and H-beta zeolite than HZSM-5. So in this report, we chose PP as carbon source in the presence of H-MMT and H-beta zeolite, and as for HZSM-5 zeolite, hexene was chosen as carbon source.

\section{Experimental}

\subsection{Materials and preparation of samples}

PP was purchased from PanJin Petrochemical Co. Ltd, with a melt flow index of $0.8 \mathrm{~g} / \mathrm{min} . \mathrm{Ni}_{2} \mathrm{O}_{3}$ was kindly pro- vided by Lingfeng Chemical Company of Shanghai. Ni-Cat (nickel supported on silica-alumina, Ni content, $\sim 66 \%$ ), and 1-hexene came from Alfa Aesar. OMMT (Cloisite 15 A) was purchased from Southern Clay, modifier concentration: 125 meq/100 g clay. Commercial HZSM-5 (Si/Al: 38, pore size: $0.54,0.56 \mathrm{~nm}$ and $0.51,0.55 \mathrm{~nm}$ diameters) and $\mathrm{H}-$ beta (Si/Al: 70 , pore sizes: $0.55,0.55 \mathrm{~nm}$ and $0.64,0.76 \mathrm{~nm}$

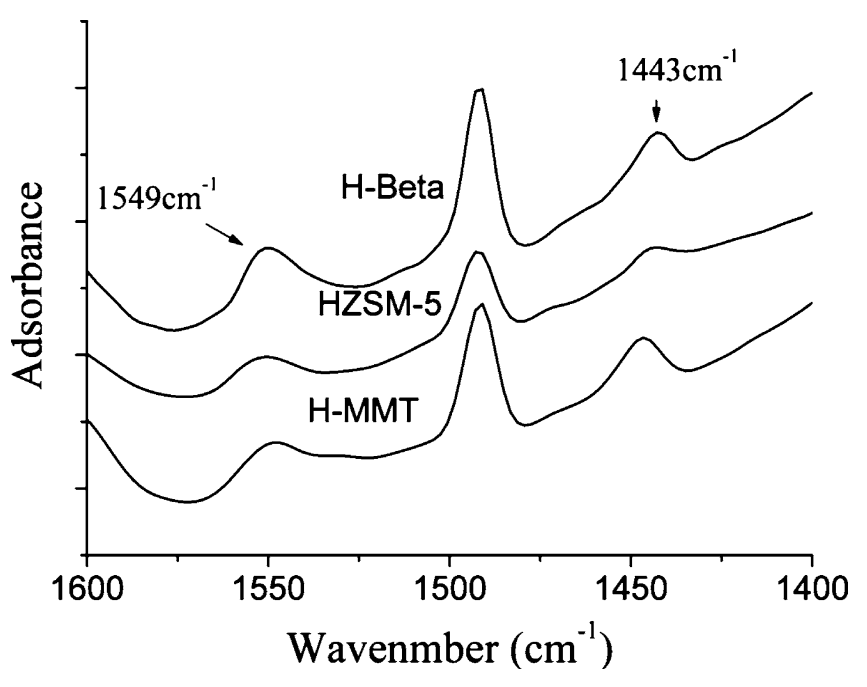

Figure 1. FTIR spectra of adsorbed pyridine on H-beta, HZSM-5 and H-MMT from OMMT in $1400-1600 \mathrm{~cm}^{-1}$ range after pyridine adsorption and then desorption at $150^{\circ} \mathrm{C}$.

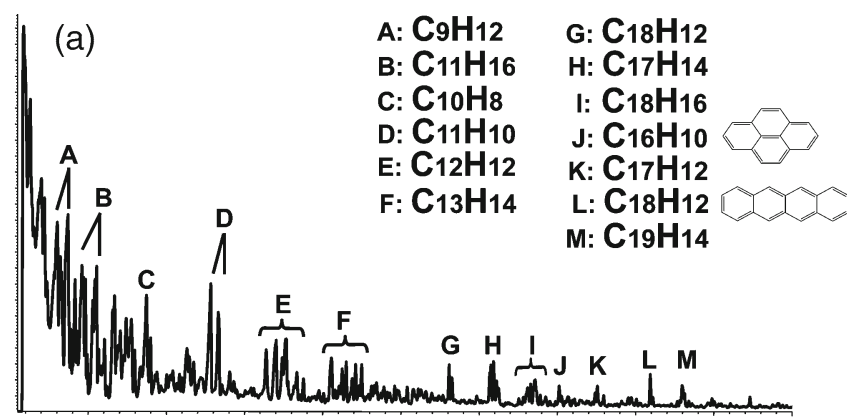

(b)

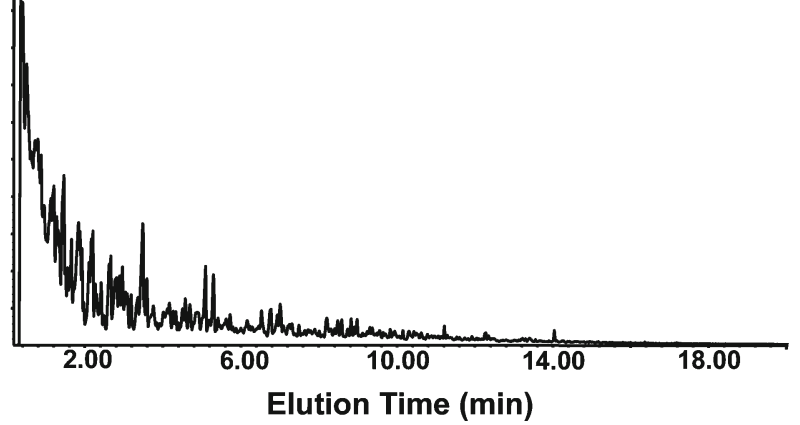

Figure 2. Gas chromatography-mass spectrometry (GC-MS) spectra of degradation products after pyrolysis: (a) $\mathrm{PP} / \mathrm{H}-\mathrm{Beta}$ and (b) $\mathrm{PP} / \mathrm{H}-\mathrm{Beta} / \mathrm{Ni}_{2} \mathrm{O}_{3}$. 
Table 1. Effect of the content of H-beta on the yield of MWCNTs and purity.

\begin{tabular}{|c|c|c|c|c|c|}
\hline $\begin{array}{l}\text { H-beta } \\
(\mathrm{wt} \%)\end{array}$ & $\begin{array}{c}\mathrm{PP} \\
(\mathrm{wt} \%)\end{array}$ & $\begin{array}{l}\mathrm{Ni}_{2} \mathrm{O}_{3} \\
(\mathrm{wt} \%)\end{array}$ & $\begin{array}{l}\text { Yield of residue } \\
\qquad(\mathrm{wt} \%)\end{array}$ & $\begin{array}{l}\text { Yield of MWCNTs } \\
\qquad(\mathrm{wt} \%)\end{array}$ & $\begin{array}{c}\text { Purity } \\
(\%)\end{array}$ \\
\hline 0 & 97 & 3 & $10 \cdot 3$ & $5 \cdot 0$ & $48 \cdot 7$ \\
\hline 1 & 96 & 3 & $20 \cdot 1$ & $12 \cdot 5$ & $62 \cdot 2$ \\
\hline 3 & 94 & 3 & $54 \cdot 6$ & $43 \cdot 2$ & $79 \cdot 1$ \\
\hline 5 & 92 & 3 & $57 \cdot 8$ & $52 \cdot 0$ & $90 \cdot 0$ \\
\hline 7 & 90 & 3 & $55 \cdot 0$ & $47 \cdot 1$ & $85 \cdot 7$ \\
\hline 9 & 88 & 3 & $56 \cdot 0$ & $46 \cdot 4$ & $82 \cdot 6$ \\
\hline
\end{tabular}
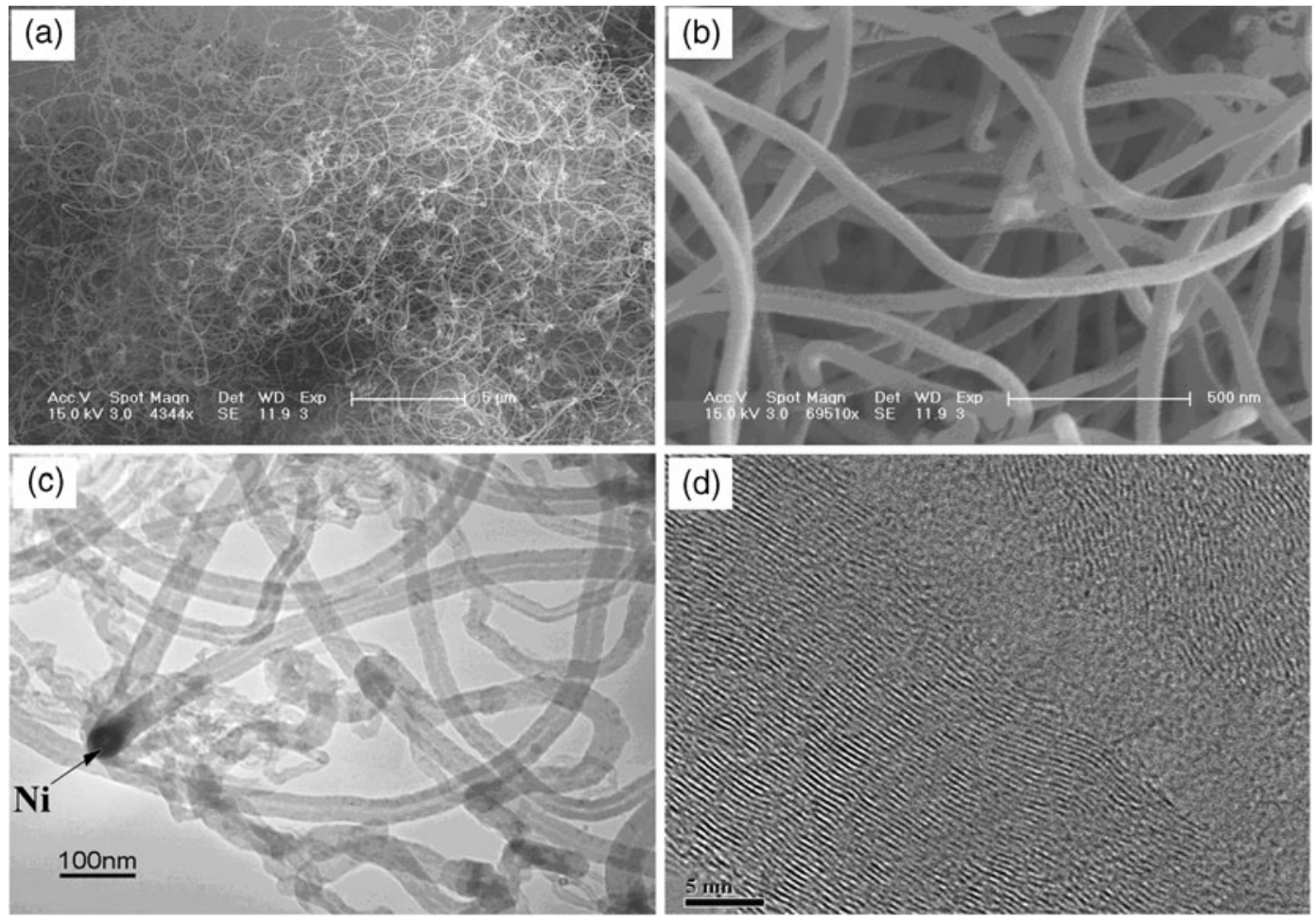

Figure 3. Microstructure of char prepared from $\mathrm{PP} / \mathrm{H}-\mathrm{Beta} / \mathrm{Ni}_{2} \mathrm{O}_{3}$ mixtures with $5 \mathrm{wt} \%$ of $\mathrm{H}-\mathrm{Beta}$ and $3 \mathrm{wt} \%$ of $\mathrm{Ni}_{2} \mathrm{O}_{3}$ via pyrolysis. (a) and (b) FE-SEM micrographs of obtained samples, (c) TEM micrograph of sample and (d) higher magnification TEM of a MWCNT.

diameters) zeolite came from Nankai University Catalyst Factory. Pyrolysis sample was prepared in a Brabender mixer at $100 \mathrm{rpm}$ and $180^{\circ} \mathrm{C}$ for $10 \mathrm{~min}$.

The device for synthesizing MWCNTs was a kind of CVD apparatus. The quartz tube, mounted in an electrical tube furnace, was heated to $700^{\circ} \mathrm{C}$ in $\mathrm{N}_{2}$ atmosphere. Subsequently, $\sim 10 \mathrm{~g}$ of the above pyrolysis sample was put into a quartz boat. After no gaseous fraction was released, the charred residue was collected and the condensed product was collected in a cold trap. For 1-hexene, it was injected into the quartz tube from one end. Ni-Cat was placed in the bottom of quartz tube. The charred residue obtained from the above process was purified with hydrofluoric acid (HF) and nitric acid $\left(\mathrm{HNO}_{3}\right)$ to eliminate inorganic solids and amorphous carbon. HF is effective to dissolve inorganic solids. According to Monthiouz et al (2001) and Ko et al (2005), nitric acid can effectively oxidize amorphous carbon on the surface of CNTs. So in this experiment, the oxidation reaction of nitric acid could eliminate most of the amorphous carbon. The yield of the MWCNTs $(y)$ is calculated from the amount of the purified char $(M)$ divided by the amount of PP $\left(M_{\mathrm{PP}}\right)$, $y=\left(M / M_{\mathrm{PP}}\right) \times 100 \%$. The purity of MWNTs was calculated from the amount of purified char $(M)$ divided by the amount of the charred residue $(R), p=(M / R) \times 100 \%$. All of the samples were weighed by an analytical balance.

\subsection{Characterization}

Acidity testing of the above aromatization catalyst was done by pyridine adsorption (Parry 1963). In a sealed tube, about $15 \mathrm{mg}$ of the sample was exposed to the pyridine vapour 
at $150^{\circ} \mathrm{C}$ for $1 \mathrm{~h}$. Subsequently, the sample was transferred out of the pyridine vapour and kept at $150^{\circ} \mathrm{C}$ for $1 \mathrm{~h}$ to remove the physically adsorbed pyridine. The resulting product was pelletized with $\mathrm{KBr}$ and characterized by FTIR spectrometer (BRUKER Vertex 70 FTIR). OMMT were firstly calcined to form H-form MMT (H-MMT) under nitrogen atmosphere at $700^{\circ} \mathrm{C}$ before adsorption of pyridine. The morphologies of the as-prepared products were examined with field-emission scanning electron microscopy (FE-SEM, XL303SEM) and transmission electron microscopy (TEM, JEM-2010, operated at $200 \mathrm{kV}$ voltage). High-resolution transmission electron microscopy (HRTEM, Philips CM200, $200 \mathrm{kV}$ ) was used to further characterize the morphologies and microstructures of the purified product. Raman spectra (T6400, excitation beam wavelength: $514.5 \mathrm{~nm}$ ) was used to characterize the vibrational properties of the obtained MWCNTs. Based on a recent report (McKee and Vecchio 2006), TGA was carried out by means of a Perkin-Elmer TGA 7 thermoanalyser. The samples were heated in air to $300^{\circ} \mathrm{C}$ at a heating rate of $20^{\circ} \mathrm{C} / \mathrm{min}$ and allowed to equilibrate for $1 \mathrm{~min}$ before being heated from 300 to $700^{\circ} \mathrm{C}$ at $1{ }^{\circ} \mathrm{C} / \mathrm{min}$. Gas chromatography-mass spectrometry (GC-MS, AGILENT 5975 MSD) was used to measure composition of the condensed products.

\section{Results and discussion}

Figure 1 presents IR spectra of pyridine adsorption on $\mathrm{H}$ Beta, HZSM-5 and H-MMT. Upon pyridine adsorption at $150^{\circ} \mathrm{C}$, absorption bands associated with the chemisorbed pyridine were observed at 1549,1491 and $1443 \mathrm{~cm}^{-1}$. According to previous reports on IR studies in pyridine chemisorption (Parry 1963), the pyridine band at $1549 \mathrm{~cm}^{-1}$ can be assigned to pyridinium ions $(\mathrm{C}-\mathrm{C}$ bands of pyridine adsorbed) which are characteristic of Brønsted acid sites, and

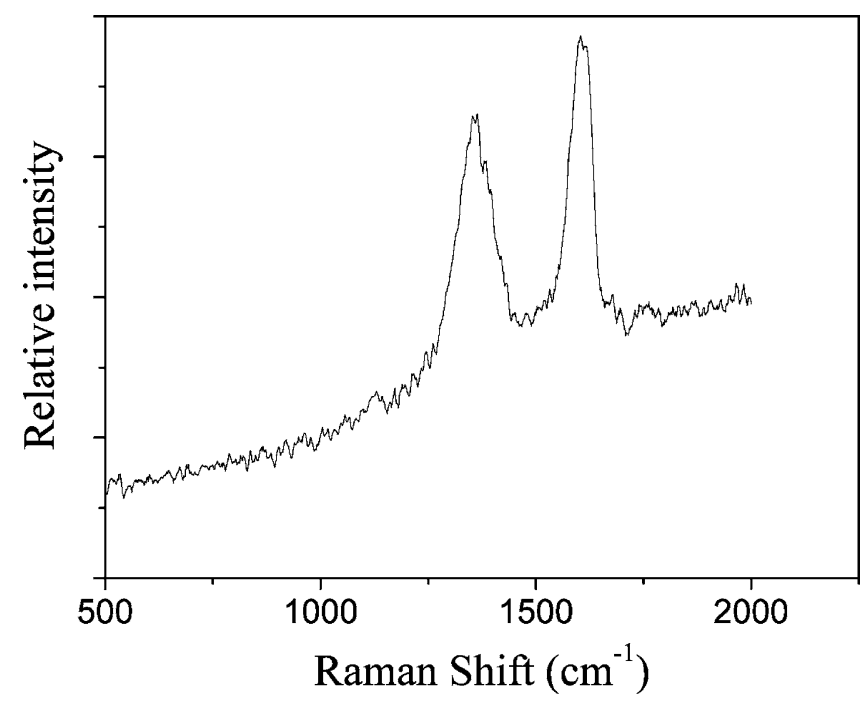

Figure 4. Raman spectra of purified MWCNTs from $\mathrm{PP} / \mathrm{H}-$ Beta/ $\mathrm{Ni}_{2} \mathrm{O}_{3}$ with $5 \mathrm{wt} \%$ of $\mathrm{H}$-Beta and $3 \mathrm{wt} \%$ of $\mathrm{Ni}_{2} \mathrm{O}_{3}$.

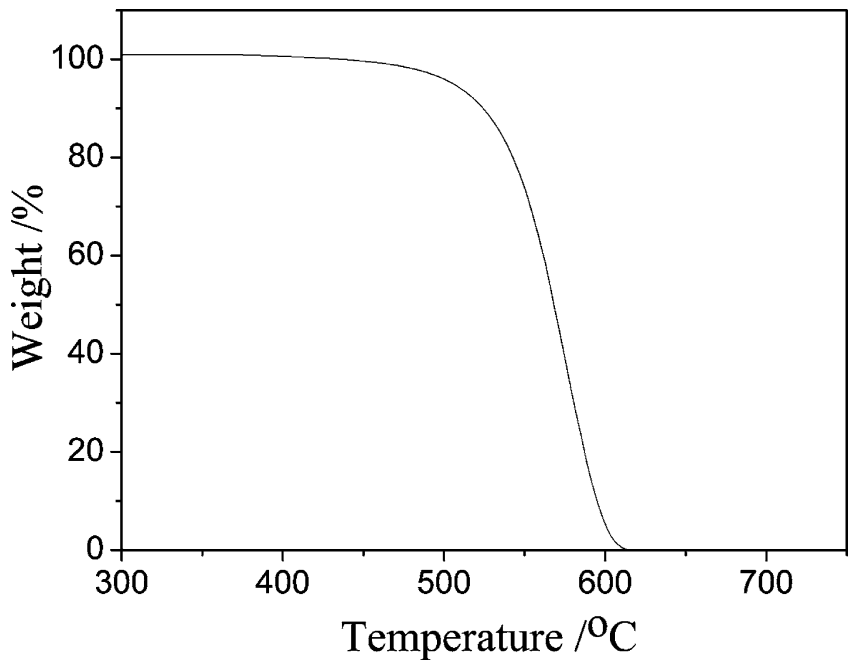

Figure 5. TGA weight loss curves of purified MWCNTs from $\mathrm{PP} / \mathrm{H}-\mathrm{Beta} / \mathrm{Ni}_{2} \mathrm{O}_{3}$ with $5 \mathrm{wt} \%$ of $\mathrm{H}-$ Beta and $3 \mathrm{wt} \%$ of $\mathrm{Ni}_{2} \mathrm{O}_{3}$.

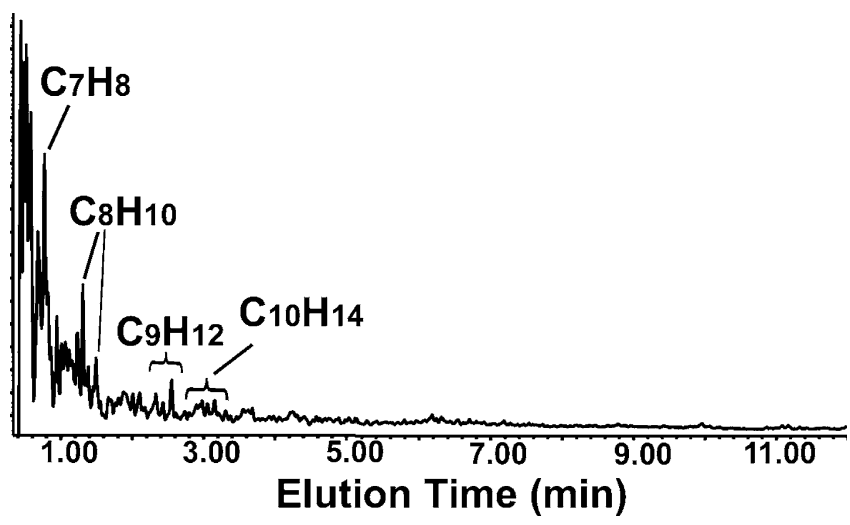

Figure 6. Gas chromatography-mass spectrometry (GC-MS) spectra of degradation products of PP/OMMT after pyrolysis.

the band at $1443 \mathrm{~cm}^{-1}$ to Lewis acid-coordinated pyridine (C-C bands of pyridine adsorbed). The band at $1491 \mathrm{~cm}^{-1}$ consisted of bands due to the Brønsted and Lewis acid sites. Due to the concentrations of both samples in $\mathrm{KBr}$ pellets being the same (zeolite/KBr: 1/150, wt/wt), the intensity of characteristic absorption peaks in FTIR spectra can be studied for comparison to indicate the acidity relatively. It is evident that the acidity of H-Beta and H-MMT is stronger than that of H-ZSM-5 in this system.

In order to demonstrate that the aromatization reaction of PP can be catalyzed by H-Beta zeolite under high temperature condition, the condensed product collected during the pyrolysis of $\mathrm{PP} / \mathrm{H}-\mathrm{Beta}$ sample was analysed by using GCMS technique. From figure 2a, it can be seen that larger amount of aromatics such as benzene, toluene, dimethylbenzene, naphthalene and PAHs exist in the condensed product, which indicates that the H-Beta zeolite is an effective catalyst to catalyze aromatization reaction of these degradation products from PP under high temperature condition. 
Table 2. Effect of the content of OMMT on the yield of MWCNTs and purity.

\begin{tabular}{lcccr}
\hline $\begin{array}{l}\text { OMMT } \\
(\mathrm{wt} \%)\end{array}$ & $\begin{array}{c}\mathrm{PP} \\
(\mathrm{wt} \%)\end{array}$ & $\begin{array}{c}\mathrm{Ni}_{2} \mathrm{O}_{3} \\
(\mathrm{wt} \%)\end{array}$ & $\begin{array}{c}\text { Yield of residue } \\
(\mathrm{wt} \%)\end{array}$ & $\begin{array}{c}\text { Yield of MWCNTs } \\
(\mathrm{wt} \%)\end{array}$ \\
\hline 0 & 97 & 3 & $10 \cdot 3$ & $5 \cdot 0$ \\
$(\%)$
\end{tabular}
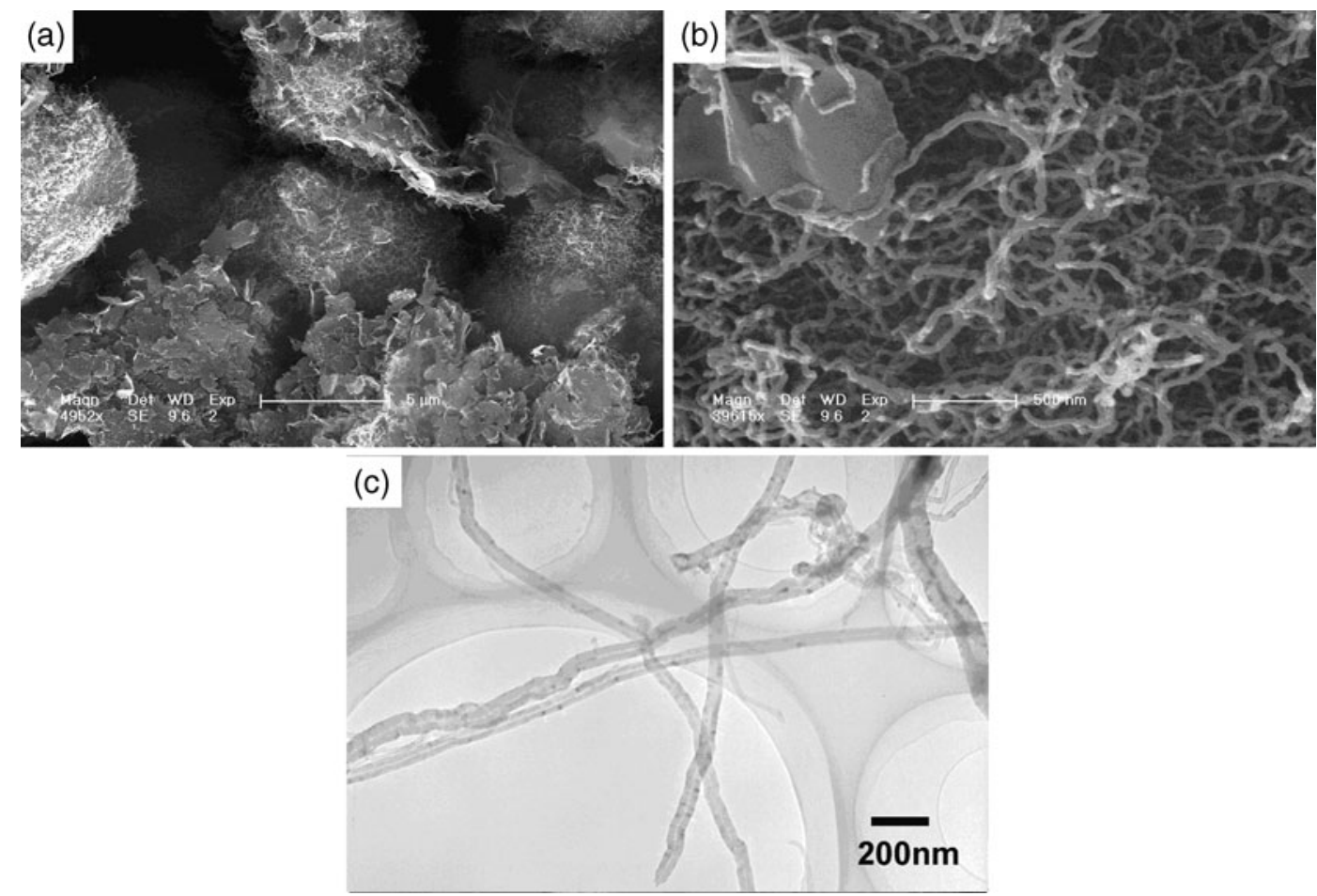

Figure 7. Microstructure of char prepared from $\mathrm{PP} / \mathrm{OMMT} / \mathrm{Ni}_{2} \mathrm{O}_{3}$ mixtures with $9 \mathrm{wt} \%$ of OMMT and $3 \mathrm{wt} \%$ of $\mathrm{Ni}_{2} \mathrm{O}_{3}$ via pyrolysis. (a) and (b) FE-SEM micrographs of obtained sample and (c) TEM micrograph of sample.

As an effective aromatization catalyst, effect of the concentration of $\mathrm{H}$-Beta in $\mathrm{PP} / \mathrm{H}-\mathrm{Beta} / \mathrm{Ni}_{2} \mathrm{O}_{3}$ composite on the yield of MWCNTs was investigated. The content of $\mathrm{Ni}_{2} \mathrm{O}_{3}$ was fixed at $3 \%$ concentration in all composites. From table 1, it can be seen that the yield of MWCNTs produced by $\mathrm{PP} / \mathrm{H}-\mathrm{Beta} / \mathrm{Ni}_{2} \mathrm{O}_{3}$ increases dramatically with the increase of the concentration of $\mathrm{H}$-Beta in the composite. The highest yielded value in these experiments was $52 \%$ when the content of H-Beta was $5 \mathrm{wt} \%$. However, when the content of $\mathrm{H}$-Beta in the composite was increased further, the yield of MWCNTs from PP/H-Beta/ $\mathrm{Ni}_{2} \mathrm{O}_{3}$ decreased a little. More interestingly, the change of MWNT purity with loading of H-beta has a similar trend with that of MWCNT yield, the highest value of purity was $90 \%$ with 5 wt $\%$ loading of H-Beta.

The char residue of $\mathrm{PP} / \mathrm{H}-\mathrm{Beta} / \mathrm{Ni}_{2} \mathrm{O}_{3}(92: 3: 5)$ was chosen to characterize the property of CNTs. The SEM images given in figure $3 \mathrm{a}$ and $\mathrm{b}$ show that the as-prepared products contain an abundance of hair-like fibres. TEM observation confirms that the hair-like fibres are MWCNTs with hollow centres, and the outer diameter of fibres is almost $50 \mathrm{~nm}$ which could be attributed to the particle size of Ni catalyst derived from $\mathrm{Ni}_{2} \mathrm{O}_{3}$ (figure $3 \mathrm{c}$ ). The detailed structure of a single tube of the as-prepared MWCNTs is given in an HRTEM image (figure 3d) which shows that it consists of graphite fringes without perfectly concentric structure.

Raman spectroscopy was used to characterize graphitization of the purified product (figure 4). The peak at $1598 \mathrm{~cm}^{-1}$ (G-band) corresponds to an $E 2 g$ mode of hexagonal graphite and is related to the vibration of $s p^{2}$-hybridized carbon atoms in a graphite layer. The $D$-band at about $1359 \mathrm{~cm}^{-1}$ is associated with the vibration of carbon atoms with dangling bonds in the plane terminations of disordered graphite or glassy carbons (Rao et al 1997). The curve shown in figure 4 

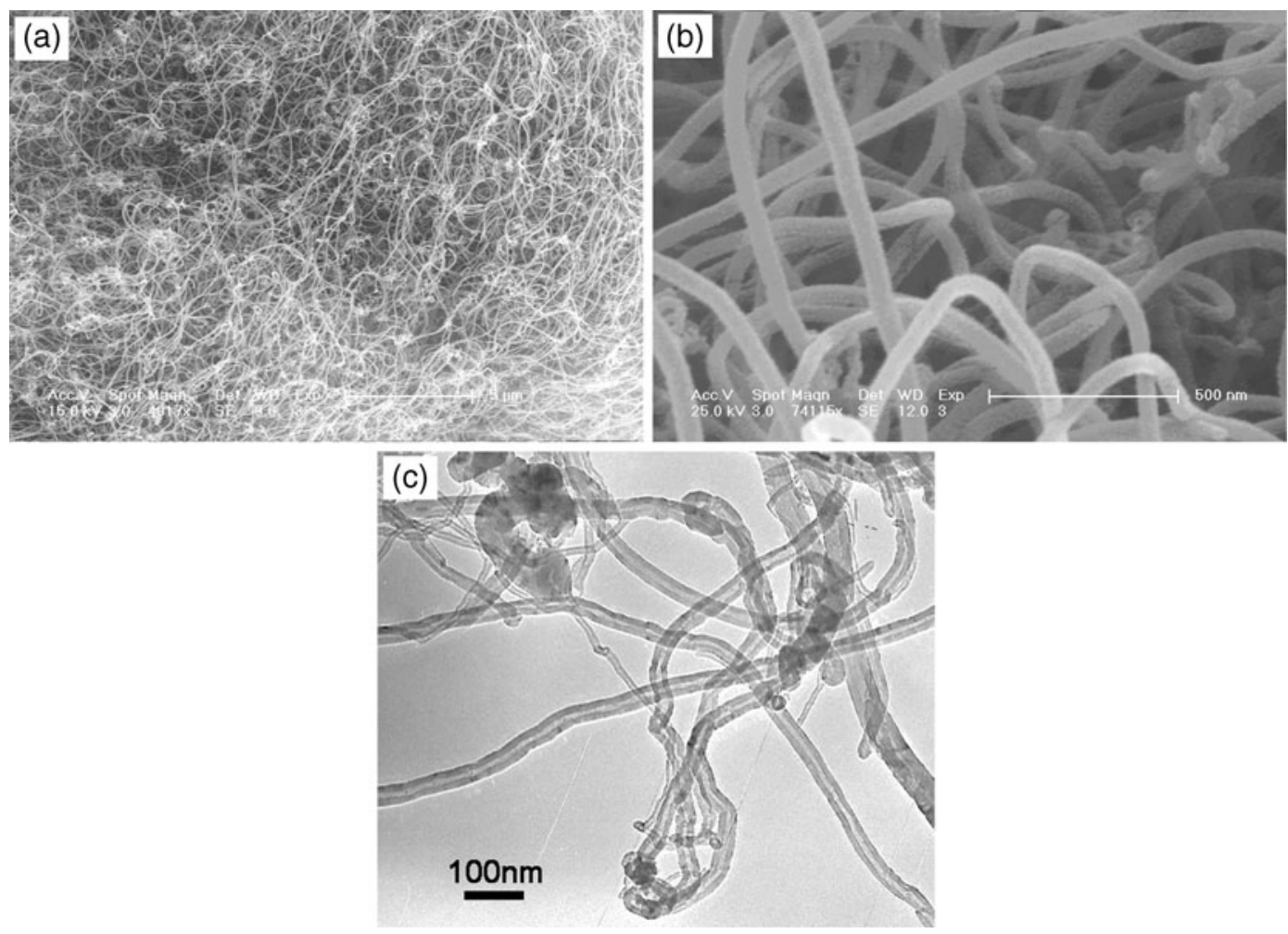

Figure 8. Microstructure of char prepared from 1-hexene with $0.1 \mathrm{~g}$ HZSM-5 and $0.05 \mathrm{~g}$ Ni-Cat via pyrolysis. (a) and (b) FE-SEM micrographs of obtained sample and (c) TEM micrograph of sample.

Table 3. Effect of combined catalysts on the MWCNT yield from 1-hexene in the pyrolyzer at $700^{\circ} \mathrm{C}$.

\begin{tabular}{lccc}
\hline & HZSM-5/g & Ni-Cat /g & Yield of MWCNTs (wt $\%)$ \\
\hline Case-1 & & 0.5 & $8 \cdot 0$ \\
Case-2 & $1 \cdot 0$ & 0.5 & $0 \cdot 0$ \\
Case-3 & $1 \cdot 0 \rightarrow$ & 0.5 & $11 \cdot 0$ \\
Case-4 & $1 \cdot 0+$ & $36 \cdot 0$ \\
\hline
\end{tabular}

Case-1: 1-hexene passed through Ni-Cat, Case-2: 1-hexene passed through HZSM-5, Case-3: 1-hexene passed through HZSM-5 and then Ni-Cat, Case-4: 1-hexene passed through the mixture of HZSM-5 and Ni-Cat.

proves that high quality of MWCNTs can be obtained through purification.

TGA was used to further evaluate graphitization of the purified MWCNTs (figure 5). The maximum weight loss occurred at $590^{\circ} \mathrm{C}$. At the same time, the purified MWCNTs decomposed a little when the temperature was below $500^{\circ} \mathrm{C}$, which indicates that the MWCNT sample is relatively pure and of high quality.

In order to confirm the relationship between the aromatization reaction of PP and the formation of MWCNTs from PP, we further compared the condensed product of $\mathrm{PP} / \mathrm{H}-\mathrm{Beta}$ and $\mathrm{PP} / \mathrm{H}-\mathrm{Beta} / \mathrm{Ni}_{2} \mathrm{O}_{3}$. From figure $2 \mathrm{~b}$, it can be seen that almost all the aromatics content in the liquid product decrease and even some aromatics with higher carbon numbers disappear when $\mathrm{Ni}_{2} \mathrm{O}_{3}$ was added into the $\mathrm{PP} / \mathrm{H}-$ Beta composite. It indicates that the aromatization action of H-Beta on carbon source has been used to improve the formation of CNTs due to the combination of H-Beta and $\mathrm{Ni}_{2} \mathrm{O}_{3}$ catalysts.

Same phenomenon occurred, when the PP/OMMT composite was decomposed via pyrolysis in nitrogen. From figure 6 , it can be seen that larger amount of aromatics appear in the condensed product. At the same time, the OMMT content in the PP/OMMT/ $/ \mathrm{Ni}_{2} \mathrm{O}_{3}$ composite has the same effect on the yield and purity of MWCNTs with H-Beta in $\mathrm{PP} / \mathrm{H}-\mathrm{Beta} / \mathrm{Ni}_{2} \mathrm{O}_{3}$ (table 2). The MWCNTs produced by it was also characterized by SEM and TEM as shown in figure 7. 


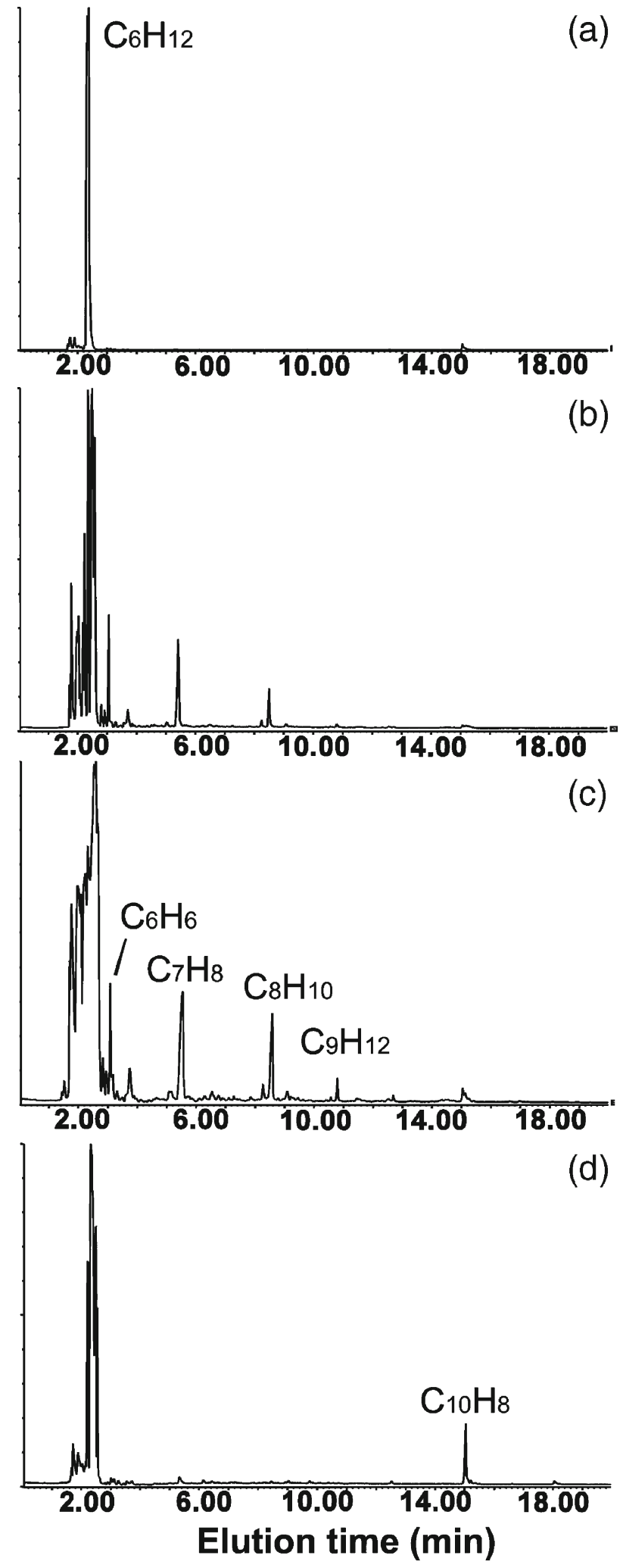

Figure 9. Gas chromatography-mass spectrometry (GC-MS) spectra of condensed products via pyrolysis 1-hexene. (a) $\mathrm{Ni}$ Cat (pass through Ni-Cat), (b) HZSM-5 (pass through HZSM-5), (c) HZSM-5 $\rightarrow$ Ni-Cat (pass through HZSM-5 and then Ni-Cat) and (d) Ni-Cat + HZSM-5 (pass through mixture of HZSM-5 and Ni-Cat).
As for HZSM-5, its aromatization effectiveness had been demonstrated in many previous works (Sun and Zhao 1991; Shu et al 1999; Barthos et al 2006). Here we choose CVD to synthesize CNTs. 0.05 g Ni-Cat and 0.1 g HZSM-5 were first mixed and put in a quartz tube, then the temperature of quartz tube was raised to $700^{\circ} \mathrm{C}$, after that, $20 \mathrm{ml}$ of 1 -hexene was added in $1 \mathrm{~min}$. Almost $1.87 \mathrm{~g}$ as-prepared product was obtained, which was characterized by SEM (figures 8a, b). After purification, the MWCNTs $(1.70 \mathrm{~g})$ were further characterized by TEM (figure $8 \mathrm{c}$ ). From the SEM images of low magnification, we can clearly see that abundance of hair-like fibres exist in the product. TEM images demonstrated that the hair-like fibres were MWCNTs with hollow centres. The outer diameter of a typical MWCNT was almost $50 \mathrm{~nm}$. High yield formation of MWCNTs can be ascribed to the aromatization reaction of 1hexene catalyzed by HZSM-5 when it came into contact with Ni-Cat.

In order to confirm above assumption, the following experiments were designed: case-1, only $\mathrm{Ni}$-Cat was put within the pyrolyzer at $700^{\circ} \mathrm{C}$, then $20 \mathrm{ml}$ of 1 -hexene was added in 1 min; case-2, only HZSM-5 was put within the pyrolyzer at $700^{\circ} \mathrm{C}$, then $20 \mathrm{ml}$ of 1 -hexene was added in $1 \mathrm{~min}$; case-3, Ni-Cat and HZSM-5 were separately put within the pyrolyzer at $700^{\circ} \mathrm{C}$, then $20 \mathrm{ml}$ of 1 -hexene was added in $1 \mathrm{~min}$, which passed through HZSM-5 and then Ni-Cat; case4, after Ni-Cat and HZSM-5 were first mixed together, the mixture was put within the pyrolyzer at $700^{\circ} \mathrm{C}$, then $20 \mathrm{ml}$ of 1-hexene was added in $1 \mathrm{~min}$. The composition of catalyst and yield of MWCNTs are listed in table 3. The results show that in case-4, the highest MWCNT yield was achieved, which further demonstrated our previous assumption. The liquid of the product was measured by GC-MS. In figure 9, it can be seen that almost no aromatics was produced when only Ni-Cat was put within the pyrolyzer. On the contrary, much of the aromatics appeared when only HZSM-5 existed. However, when both Ni-Cat and HZSM-5 were separately put within the pyrolyzer, the concentration of some aromatics increased. The amount of as prepared product increased a little. The result indicates that aromatics are not easy to be converted directly to MWCNTs in conventional CVD. The increase of the amount of aromatics can be attributed to the dehydrogenization of nickel catalyst. Surprisingly, when NiCat and HZSM-5 were mixed together within the pyrolyzer in case-4, the concentration of some aromatics decrease and even some aromatics with higher carbon numbers disappear in condensed product. At the same time, the amount of as prepared product increased a lot. Because many of the aromatics were not easy to be converted directly to MWCNTs which has been demonstrated in case-3, the aromatization reaction of the hydrocarbons should directly accelerate the formation of MWCNTs in case-4, instead of converting to aromatics then to MWCNTs through two steps.

The mechanism aspect is depicted in figure 10. If only the aromatization catalyst existed in our systems, the carbon source in pyrolyzer will only pass the surface of aromatization catalyst, and then aromatized by it. Dehydrogenation- 


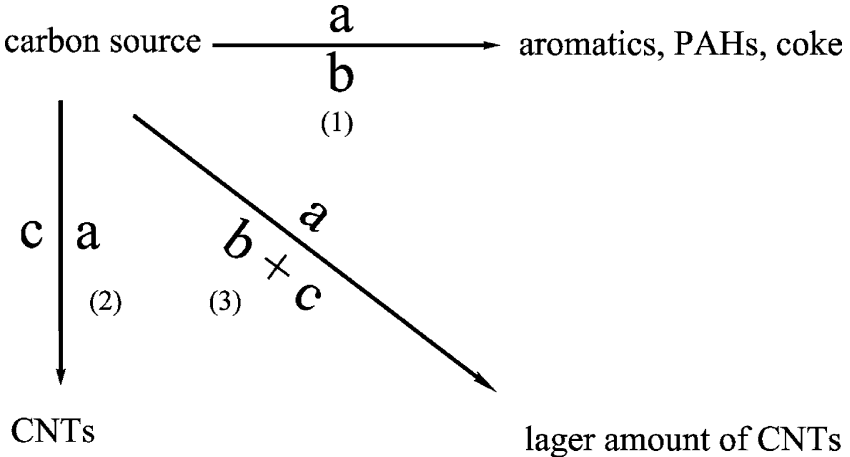
a: dehydrogenation-cyclization, condensation
b: aromatization catalyst
c: $\mathrm{Ni}$

Figure 10. Diagram of synergistic effect of aromatization catalyst in synthesis of CNTs using Ni as catalyst.

cyclization and condensation reaction belong to the necessary steps to form aromatics (reaction 1 ). If only $\mathrm{Ni}$ catalyst is present in our systems, the carbon source in pyrolyzer will only pass the surface of $\mathrm{Ni}$ catalyst, and then carbonized by it to form CNTs. Similarly, the dehydrogenation-cyclization and condensation reaction also happens on the surface of $\mathrm{Ni}$ catalyst (reaction 2). However, when the Ni catalyst is combined with the aromatization catalyst in our systems, the process of dehydrogenation-cyclization and condensation reaction of the carbon source on the surface of Ni catalyst for the formation of CNTs will be accelerated by the aromatization catalyst (reaction 3). Here, it has been demonstrated that the aromatization catalysts, H-Beta zeolite, HZSM-5 and OMMT all can effectively enhance the yield of MWCNTs from PP or 1-hexene.

\section{Conclusions}

It is demonstrated that aromatization reaction of carbon source can effectively promote CNTs formation during the process of synthesizing CNTs. The reason analyzed is that the aromatization catalyst can accelerate the dehydrogenation-cyclization and condensation reaction which belong to the necessary steps for the formation of CNTs. It is not difficult to imagine that the synergistic effect of the aromatization catalyst with nickel catalyst take place not only in this process, but also in other processes for synthesizing CNTs. At the same time, it will be helpful to better understand the mechanism about the formation of CNTs, direct the efforts for industrializing the synthesis process, and produce CNTs with higher quality for materials research and commercial exploitation. Owing to not completely demonstrating some detailed steps by which aromatization reac- tion of carbon source effectively promotes the formation of CNTs, further researches are on the way in our laboratory.

\section{References}

Ajayan P M 1999 Chem. Rev. 991787

Ajayan P M and Ebbesen T W 1997 Rep. Prog. Phys. 60 1025

Avouris Ph 2002 Chem. Phys. 281429

Barthos R, Szechenyi A and Solymosi F 2006 J. Phys. Chem. B110 21816

Dillon A C and Heben M J 2001 Appl. Phys. A72 133

Dresselhaus M S, Dresselhaus G and Eklund P C 1996 Science of fullerenes and carbon nanotubes (New York: Academic Press) pp 16-17

Ebbesen T W 1997 Carbon nanotubes: preparation and properties (Boca Raton, FL: CRC Press) pp 296-297

Height M J, Howard J B, Tester J W and Vander Sande J B 2005 J. Phys. Chem. B109 12337

Iijima S 1991 Nature 35456

Kiang C H, Goddard W A, Beyers R and Bethune D S 1995 Carbon 33903

Ko F H, Lee C Y, Ko C J and Chu T C 2005 Carbon 43727

Landi B J, Raffaelle R P, Heben M J, Alleman J L, Van Derveer W and Gennett T 2002 Nano Lett. 21329

Li W Z et al 2002 Carbon 40787

McKee G S B and Vecchio K S 2006 J. Phys. Chem. B110 1179

Monthiouz M, Smith B W, Burteaux B, Claye A, Fischer J E and Luzzi D E 2001 Carbon 391251

Parry E P 1963 J. Catal. 2371

Rao A M et al 1997 Science 275187

Reilly P T A and Whitten W B 2006 Carbon 441653

Reilly P T A, Gieray R A, Whitten W B and Ramsey J M 2000a Combust. Flame $\mathbf{1 2 2} 90$

Reilly P T A, Gieray R A, Whitten W B and Ramsey J M 2000b J. Am. Chem. Soc. 12211596

Rodgers R P, Reilly P T A, Whitten W B and Ramsey J M 2003 Carbon 411469

Rodgers R P, Reilly P T A, Whitten W B and Ramsey J M 2004 Chem. Phys. Lett. 397324

Saito R, Dresselhaus M S and Dresselhaus G 1998 Physical properties of carbon nanotubes (New York: World Scientific) pp 24-25

Shu J, Adnot A and Grandjean B P A 1999 Ind. Eng. Chem. Res. 38 3860

Song R J et al 2007 Chem. Eur. J. 133234

Sun D L and Zhao Z G 1991 Ind. Eng. Chem. Res. 302013

Stein S and Fahr A 1985 J. Phys. Chem. 893714

Tang T, Chen X C, Chen H, Meng X Y, Jiang Z W and Bi W G 2005a Chem. Mater. 172799

Tang T, Chen X C, Meng X Y, Chen H and Ding Y P 2005b Angew. Chem. Int. Ed. 441517

Thostenson E T, Ren Z F and Chou T W 2001 Compos. Sci. Technol. 611899

Wood J R et al 2000 Phys. Rev. B62 7571

Yakobson B I and Smalley R E 1997 Am. Sci. 7-8 324 